a periodicity similar to that occurring in the head movement records of W. D. W. From a study of several of her steadiest records, it was thus possible to deduce her head movements indirectly, as shown in Fig. 2.

Records 3 and 4 show typical eye movements for each subject during monocular fixation, when the subject was at his steadiest. The main feature which distinguishes the eye movements from the head movements is the rapid flick lasting $0.02-0.03$ sec. and indicating a momentary angular movement of the eye of amplitude ranging from about 3 to 14 minutes of are, and an average frequency of about 2 per sec. for W. D. W. and 3 every 2 sec. for M. P. L.

Between these flicks most of the recorded movement could be attributed to head movements, although in the absence of simultaneous head and eye movement records this is not certain. After each flick, the records suggest that the eye may take up a slightly different fixation direction displaced some fow minutes of arc from the previous direction. This effect might be due to slight irregularities building up on the corneal surface; however, after-image experiments, for example, observation of the after. image of a bright annulus during fixation of a point source of light, seem to confirm the first interpretation.

On the majority of occasions, however, it was impossible to obtain records as steady as those in Figs. 3 and 4, random eye movements of 10 or 20 minutes of arc commonly occurring, as shown in Figs. 5 and 6 . The gap at $P$ in record 5 is due to a blink and indicates obstruction at the centre of the cornea lasting for 0.2 sec., in general agreement with data presented recently by Lawson ${ }^{2}$; in this, as in most cases, little subsequent disturbance in fixation direction has been produced.

Tests made with various fixation targets showed no significant variation in fixation ability, relative to the day-to-day variation in abirity to fixate on a given target. There is little evidence of any fine vibratory movements having a period of the order of 0.02 sec. as reported by Adler and Fliegelman ${ }^{3}$ and discussed at length by Jones and Higgins ${ }^{4}$ in connexion with the resolving power of the eye.

We wish to acknowledge with thanks the financial support of the Medical Research Council in this work, and also the help of Mr. J. Baird in connexion with the dental regulation band.

$$
\text { MARY P. LORD }
$$$$
\text { W. D. WRIGHT }
$$

Technical Optics Section,

Imperial College of Science and Technology, London, S.W.7.

$$
\text { March } 12 .
$$

1 Lord, Proc. Phys. Soc. (in course of publication).

${ }^{2}$ Lawson, Nature, 161, 154 (1948).

- Adler and Fliegelman, Arch. Ophthalmol., 12, 475 (1934).

‘ Jones and Higgins, J. Opt. Soc. Amer., 37, 217 (1947).

\section{The Fundamental Colour Sensations}

IF pressure, sufficient to be thoroughly uncomfort. able, is maintained by the fingers on the eyeball, numerous violet dots will eventually appear in among the other entoptical phenomena. Each dot lasts a second or so. If the pressure is suddenly released, all the entoptical phenomena disappear except the violet dots, which are left for a few seconds against a dark background. They are fairly uniformly distributed over the field of view, and may number anything up to, say, 100. From the fact that all the violet dots are of exactly the same hue, saturation and intensity and of approximately the same size, one is probably justified in assuming that they are the fundamental violet sensation. By looking at a spectrum with the other eye (light-adapted) it is possible to match their hue against a spectral hue. They match $440.5 \mathrm{~m} \mu$, the standard deviation of each observation being $\pm 5 \mathrm{~m} \mu$ and that of the mean being $\pm 0.5 \mathrm{~m} \mu$. They are considerably more saturated in colour than spectral violet. Their apparent angular diameters are of the order of $10^{\prime}$. From the tritanopic isocolour chart given by Pitt ${ }^{1}$, which depends on a description by König of five pathological cases of tritanopia, one can deduce that the fundamental violet sensation is equivalent to the sensation produced by $435 \mathrm{~m} \mu$, except that it is more saturated. Considering the inadequacy of the data available to Pitt, the discrepancy between $440.5 \mathrm{~m} \mu$ and $435 \mathrm{~m} \mu$ is not significant.

Gentler pressure will, in suitable circumstances, produce a uniform green colour of low intensity throughout the field of view. If one continues with this pressure for a minute or so, a green patch of greater luminosity but of about the same saturation appears, usually in the temporal field. By suitable adjustment of the pressure, it may be made to grow into a broad elliptical halo surrounding the macula, which by comparison looks black. The horizontal axes of the halo are about $1 \cdot 3$ times the vertical, the inside horizontal axis subtending about $7^{\circ}$ and the outside about $30^{\circ}$. Both measurements are very variable, and occasionally the hole in the centre of the halo is green and is surrounded by darkness. The halo is slightly more saturated than any spectral green and matches $555 \mathrm{~m} \mu$, the standard deviation of each observation being $3 \mathrm{mu}$ and that of the mean being $0.7 \mathrm{~m} \mu$. This does not agree with the value $505 \mathrm{~m} \mu$ obtained from Pitt's colour triangle for the hue of the green fundamental sensation, nor is it sufficiently saturated. It seems, indeed, to be a most improbable value for a fundamental sensation on any trichromatic theory which disallows negative stimulation. As the halo gets fainter, it appears to become slightly bluer in the same way as spectral $555 \mathrm{~m} \mu$ does, but it is doubtful whether it ever becomes as blue as $505 \mathrm{~m} \mu$. Hence, in terms of trichromatic theory, it is probably a mixture in fairly constant proportions of the red and the green funda. mental sensations only.

Reddish spots can also be made to appear. They are apparently of two or more different kinds, the smallest being very much smaller than the violet dots. All types are so fleeting that it is difficult to match them against the spectrum, or even to see whether they are alike.

No colourless entoptical phenomena appear as a consequence of pressure. This result and the occurrence of the violet dots at approximately the position in the eolour triangle predicted by Pitt are rather more readily explained on classical trichromatic theory than on Granit's $s^{2}$ theory of dominators and modulators.

All the above results were obtained on one observer (male) with normal colour vision.

\section{A. H. S. HolbourN}

Department of Natural Philosophy, Marischal College,

Aberdeen.

March 3.

\footnotetext{
${ }^{1}$ Proe. Roy. Soc., B, 132, 101 (1945) ; chart on p. 107 wrongly labelled protanopic.

s"Sensory Mechanisms of the Retina" (Oxford Univ. Press).
} 